Bibliotecas. Vol 39, № 2, julio - diciembre, 2021. EISSN: 1659-3286

URL: http://www.revistas.una.ac.cr/index.php/bibliotecas/index

DOI: http://dx.doi.org/10.15359/rb.39-2.2

Licencia: Creative Commons (BY-NC-SA) 4.0 Internacional

\title{
Olinta Ariosa Morales: precursora de la bibliotecología y las bibliotecas en Cuba
}

\author{
Olinta Ariosa Morales: Precursor of Librarianship and Libraries in Cuba
}

\author{
Miriam Caridad Ruíz García \\ Universidad Central "Marta Abreu" de Las Villas, Cuba. \\ ORCID: https://orcid.org/0000-0002-5309-6160 \\ Luis Ernesto Paz Enrique \\ Universidad Central "Marta Abreu" de Las Villas, Cuba. \\ ORCID: https://orcid.org/0000-0001-9214-3057 \\ Cándida Judith Guerra Miranda \\ Biblioteca Provincial "Marti" de Villa Clara, Cuba \\ ORCID: https://orcid.org/0000-0002-6150-8583
}

Recibido: 13 de noviembre de 2020

Aceptado: 18 de mayo de 2021

Publicado: 21 de junio de 2021

\begin{abstract}
Resumen
Olinta Ariosa Morales constituye una personalidad relevante en el ámbito bibliotecológico cubano. Tuvo una amplia labor al frente de varias responsabilidades que moldearon la estructura que posee la bibliotecología contemporánea cubana. Ante la ausencia de investigaciones dedicadas a la personalidad, se planteó como objetivo establecer las contribuciones realizadas a la bibliotecología cubana por la Dra. Olinta Ariosa Morales. La investigación se clasificó como histórica y se desarrolla desde una perspectiva cualitativa. Se seleccionó el método biográfico para su implementación. Para la obtención de resultados se emplearon métodos en los niveles teórico y empírico, fundamentalmente la historia de vida. Destacó el empleo de la técnica de la entrevista a profundidad, la cual fue aplicada a colegas, familiares y estudiosos de la vida de Olinta. Se obtiene como principales conclusiones que una vez graduada de Doctora en Filosofía y Letras, Olinta trabajó como auxiliar de bibliotecario en la Universidad Central "Marta Abreu" de Las Villas. Entre los años 1959 - 1999 Olinta se desempeñó como bibliotecaria y directiva en diversas instituciones, dentro de las que destaca su nombramiento como directora de la Biblioteca Nacional "José Martí" de Cuba. Fue la primera presidenta de la directiva de Asociación Cubana de Bibliotecarios. En honor a toda su labor en el ámbito de la bibliotecología se instaura en Cuba el 14 de abril como Día del Bibliotecario Escolar en honor al nacimiento de Olinta y en 2004 se crea el "Premio Nacional Olinta Ariosa Morales" para reconocer a los bibliotecarios escolares.
\end{abstract}


Palabras clave: Olinta Ariosa Morales, Biblioteca Escolar, Asociación Cubana de Bibliotecarios, Biblioteca Nacional "José Marti" de Cuba, Sistema Nacional de Bibliotecas Públicas de Cuba.

\begin{abstract}
Olinta Ariosa Morales constitutes a relevant personality in the Cuban library field. She had extensive work at the head of various responsibilities that shaped the structure of contemporary Cuban librarianship. In the absence of research devoted to personality, the objective was to establish the contributions made to Cuban librarianship by Ph D. Olinta Ariosa Morales. The research classified as historical and is developed from a qualitative perspective. The biographical method was selected for its implementation. To obtain results, methods were used at the theoretical and empirical levels, fundamentally life history. She highlighted the use of the in-depth interview technique, which was applied to colleagues, family members and students of Olinta's life. The main conclusions are that once Olinta graduated as a Doctor of Philosophy and Letters, she worked as a librarian assistant at the Universidad Central "Marta Abreu" de Las Villas. Between the years 1959 - 1999 Olinta worked as a librarian and director in various institutions, among which her appointment as director of the "José Martí" National Library of Cuba stands out. She was the first president of the board of directors of the Cuban Association of Librarians. In honor of all his work in the field of librarianship, it was established in Cuba on April 14, as the Day of the School Librarian in honor of the birth of Olinta and in 2004 the "Olinta Ariosa Morales National Award" was created to recognize librarians schoolchildren.
\end{abstract}

Keywords: Olinta Ariosa Morales, School Library, Cuban Association of Librarians, National Library "José Martí" of Cuba, National System of Public Libraries of Cuba.

\title{
I. Introducción
}

Olinta Ariosa Morales (1921-1999) constituye un ejemplo de profesional en el ámbito de la bibliotecología cubana. Las referencias sobre la vida y obra de esta figura brindan solamente elementos descriptivos que no llegan a profundizar en valiosas contribuciones realizadas a la bibliotecología cubana. Esta personalidad ha sido poco estudiada, aunque su labor tuvo repercusión en todo el territorio nacional y constituye una de las máximas representantes del campo informativo en Cuba.

El estudio sobre Olinta Ariosa Morales ha sido escaso y deficiente. La historia de la Bibliotecología, como la de cualquier campo del saber, se materializa por medio del pensamiento y las acciones de las personalidades que la ejercen; por ello es vital estudiar sus figuras notables. Se cuenta con antecedentes bibliográficos sobre investigaciones históricas que contribuyen a esclarecer y entender la contribución a la teoría y a la práctica del trabajo bibliotecario, de importantes personalidades cubanas que enriquecieron con sus 
aportes al desarrollo de la Bibliotecología en Cuba. Entre las investigaciones recuperadas destaca:

- "María Teresa Freyre de Andrade, precursora de la Bibliotecología moderna en Cuba". Se aborda de manera multifacética la vida y la obra de María Teresa Freyre de Andrade, así como su labor en el ámbito de las bibliotecas públicas y su papel de fundadora de la Bibliotecología moderna en el país (Montes de Oca, 2005).

- "María Villar Buceta: la primera profesora de Biblioteconomía en Cuba". La investigación es la historia de vida de esta prominente mujer donde se destacan los aportes que realizó a la formación de los profesionales de las bibliotecas en Cuba. Ofrece un repertorio organizado por las esferas de actividad de esta destacada personalidad (Rodríguez y Rivera, 2006).

- "Domingo Figarola Caneda: una personalidad de la cultura y la bibliotecología en Cuba". En el trabajo se realiza un breve esbozo de la vida y la obra del insigne intelectual cubano, analizando sus aportes al campo de la bibliografía y su labor como primer director de la Biblioteca Nacional "José Marti”" de Cuba (Silva y Rivera, 2006).

- "José Antonio Ramos, el autor del Manual de biblioeconomía y uno de los más ilustres intelectuales de la República en Cuba": Se aborda la labor intelectual multifacética de José Antonio Ramos. Se destacan sus contribuciones a la teoría y la práctica del trabajo bibliotecario y a la formación de los profesionales de este campo. Se esclarecen también las etapas de su vida, precedentes a la incorporación al mundo bibliotecológico (Rivera y Silva, 2006).

- "Fermín Peraza Sarausa, bibliógrafo y bibliotecólogo", se presentan los principales logros de la personalidad quien se considera como uno de los más eminentes bibliógrafos cubanos y director por más de un cuarto de siglo, de la Biblioteca Municipal de La Habana. Se destaca su labor como innovador y en la introducción en Cuba de las mejores experiencias de la práctica bibliotecaria de los países de avanzada en este campo. Se analiza su trabajo como editor del "Anuario Bibliográfico" y su contribución a la formación de los bibliotecarios en Cuba (Rivera y Silva, 2007).

Otros estudios recientes que abordan la historia de personalidades y medios de divulgación de la bibliotecología cubana son: Paz (2015), Paz, Hernández, Tamayo y Frías (2016), Paz (2018), Frías, Paz, Alomá, Tamayo, y Hernández (2019). Atendiendo a la 
personalidad de Olinta Ariosa se identificaron referentes bibliográficos que relatan aspectos de su vida y obra de forma aislada:

- "La mujer bibliotecaria cubana en la sociedad cubana de hoy". Se menciona Olinta Ariosa como una de las alumnas destacadas en la década de los 50 en la Facultad de Filosofía y Letras de la Universidad de la Habana, que acogió la enseñanza de la Bibliotecología bajo la égida de destacados profesores como Vicentina Acuña, María Teresa Freyre de Andrade, entre otros (Terry, 2004).

- "La biblioteca pública cubana en el período 1959-1989". Expone en las principales etapas de la biblioteca pública en Cuba. El período comprendido entre 1977 y 1989 tiene lugar la consolidación de la actividad bibliotecaria producto del trabajo de Olinta Ariosa. El autor establece que el primer cambio importante en la época de Olinta Ariosa fue la separación de la Dirección Nacional de Bibliotecas de la Biblioteca Nacional José Martí. Luego la creación de grupos metodológicos para el asesoramiento al trabajo profesional, el desarrollo de investigaciones científicas, la creación de la "Norma Cubana de Descripción Bibliográfica de Libros y Folletos" (Viciedo, 2006).

La mayor parte de los referentes encontrados estaban dedicados a presentar un listado cronológico de los acontecimientos de la vida de Olinta y se limitan a indicar en los lugares donde trabajó. Se ha comprobado que a pesar de que el nombre de Olinta Ariosa Morales es muy conocido en el mundo bibliotecario cubano, no existe una investigación que de manera integral enfoque la dimensión de su figura dentro de la Bibliotecología en Cuba. Se planteó como objetivo del estudio establecer las contribuciones realizadas a la bibliotecología cubana por la Dra. Olinta Ariosa Morales.

La dimensión de recopilación de historias de vida no es un procedimiento comúnmente empleado en las investigaciones históricas del ámbito de las Ciencias de la Información en Cuba. Su aplicación constituirá un referente primario para el estudio de importantes personalidades de este campo. Servirá como una herramienta didáctica para los estudiosos e interesados en investigaciones biográficas. Favorecerá el enriquecimiento de la fundamentación metodológica de la producción científica a partir de la combinación de varios métodos de investigación con el método biográfico como estrategia esencial en la obtención de los objetivos planteados. 


\section{Materiales y métodos}

La investigación clasifica como histórica y se desarrolla desde una perspectiva cualitativa. Se seleccionó el método biográfico para su implementación por permitir la construcción del estudio de caso que se presenta, sustentado en el enfoque conocido como historia de vida. Se emplearon métodos en los niveles teóricos: histórico - lógico y analítico - sintético, en el nivel empírico: historia de vida, encuesta y el análisis documental. Las técnicas empleadas fueron la entrevista a profundidad, revisión de documentos y la triangulación de información como vía para validar los datos obtenidos.

Para la aplicación de la entrevista a profundidad se seleccionaron las siguientes personas:

1. Dr. C. Emilio Luis Setién Quesada: Doctor en Ciencias de la Información. Setién fue alumno de Olinta Ariosa Morales, cuando en la década de 1960 cursó los estudios de Bibliotecología en la Universidad de La Habana.

2. Téc. Olivia Esther Ferrán Lopetegui: Técnico en ICT de la Academia de Ciencias de Cuba. Olivia trabajó junto a Olinta Ariosa siendo subalterna de la misma en el período comprendido entre 1972-1976, en el Instituto de Documentación e Información Científico Técnico de la Academia de Ciencias de Cuba.

3. Lic. Concepción Lorenzo Hernández (Conchita): Licenciada en Información Científico - Técnico y Bibliotecología. Conchita trabajó con Olinta Ariosa en el año 1980 en el Ministerio de Cultura, siendo subalterna de la misma; en diciembre de 1980 la Dirección Metodológica de Biblioteca Públicas radica en F y 15, Vedado.

- Familiares de Olinta Ariosa que accedieron a ser entrevistados:

4. Ramón Alberto Bravo Ariosa: hijo de Eneida Ariosa Morales, el mayor de los hermanos.

- Personas que se han dedicado al estudio de algunas de las facetas de su obra o personalidad.

5. Dr. Miguel Ángel Ferrer: Director del Centro Nacional de Información para la Educación.

6. Dra. Tania Alejo: Metodóloga del Centro Nacional de Información para la Educación.

7. Ms C. Margarita Bellas Vilariño: Subdirectora de Atención al Sistema Nacional de Bibliotecas Públicas y actual presidenta de la ASCUBI. 
La investigación constó de las siguientes etapas:

I. Etapa preliminar. Enfocada en identificar investigaciones similares que de forma total o parcial abordaran la vida y obra de Olinta Ariosa Morales.

II. Registro, transcripción y elaboración de los relatos de vida. Se trató de recopilar toda la documentación que reflejara la actividad de Olinta Ariosa a fin de luego procesarla y ubicarla en contexto histórico.

III. Diseño e interpretación. Establecer estrategias para la interpretación de los datos obtenidos. A partir del diseño de investigación histórica propuesto, se ordenan los resultados y se emiten valoraciones.

IV. Presentación y publicación de los relatos biográficos. Se trata de la elaboración del informe de investigación y de documentos derivados: ponencias, artículos científicos, entre otros.

\section{Resultados y discusión}

a. Niñez, juventud y acercamiento a la bibliotecología por parte de Olinta Ariosa Morales (1921-1958)

Olinta Ariosa Morales nació el 14 de abril de 1921 en el antiguo Municipio de Zulueta (hoy en la jurisdicción del municipio de Remedios) de la provincia de Las Villas ${ }^{1}$, en el seno de una familia de procedencia obrera. Era hija de Ramón Ariosa, natural de Zulueta y trabajador de la administración del antiguo central azucarero: San Agustín. Su madre: Percia Morales Morffi era natural de Santa Clara y se desempeñaba como maestra normalista. Del matrimonio nacieron ocho hijos: 1) Eneida la mayor, 2) Walterio, 2) José Ramón, 4) Percia, 5) Olinta, 6) María Yolanda, 7) Celsi y 8) Julio.

Su infancia transcurre en un ambiente de amor y respeto. Sus padres supieron inculcarle desde temprana edad una disciplina y una sólida formación ética, de las cuales ellos eran ejemplo. A la edad de diez años Olinta sufre un accidente al caerse de una escalera. Se fractura la cadera y debe ser sometida a una delicada operación, como consecuencia de la misma queda con una leve discapacidad. Según refiere su sobrino Ramón Alberto, recuerda a su tía Tin - como la llamaban en la familia - muy pequeña y con su andar pausado "arrastrando" un poco su pierna derecha. Olinta fue una niña feliz y muy aplicada 
en los estudios. Realizó sus estudios primarios en su pueblo natal, Zulueta, los estudios de Bachillerato los cursó en el Instituto de segunda enseñanza de Remedios, Las Villas.

Estudió en la década de 1940 la especialidad en estudios lingüísticos literarios dentro de la carrera de Filosofía y Letras en la facultad del mismo nombre de la Universidad de La Habana. Se gradúa en el año de 1945. Continúa sus estudios hasta alcanzar el título de doctora en Filosofía y Letras. Ya graduada no pudo ejercer su profesión por razones de discriminación racial y de género comunes en la época: era muy difícil para una mujer negra obtener un empleo en Zulueta.

En el año 1952 regresó Olinta a La Habana a buscar trabajo y nuevos horizontes. Se matriculó en la Escuela de Bibliotecarios que funcionaba como una escuela anexa a la Facultad donde había estudiado (llustración 1). Se gradúa de esos estudios en 1953. En ese período existían pocas bibliotecas en la capital y el país, por lo que tampoco, una vez graduada como bibliotecaria, pudo encontrar trabajo.

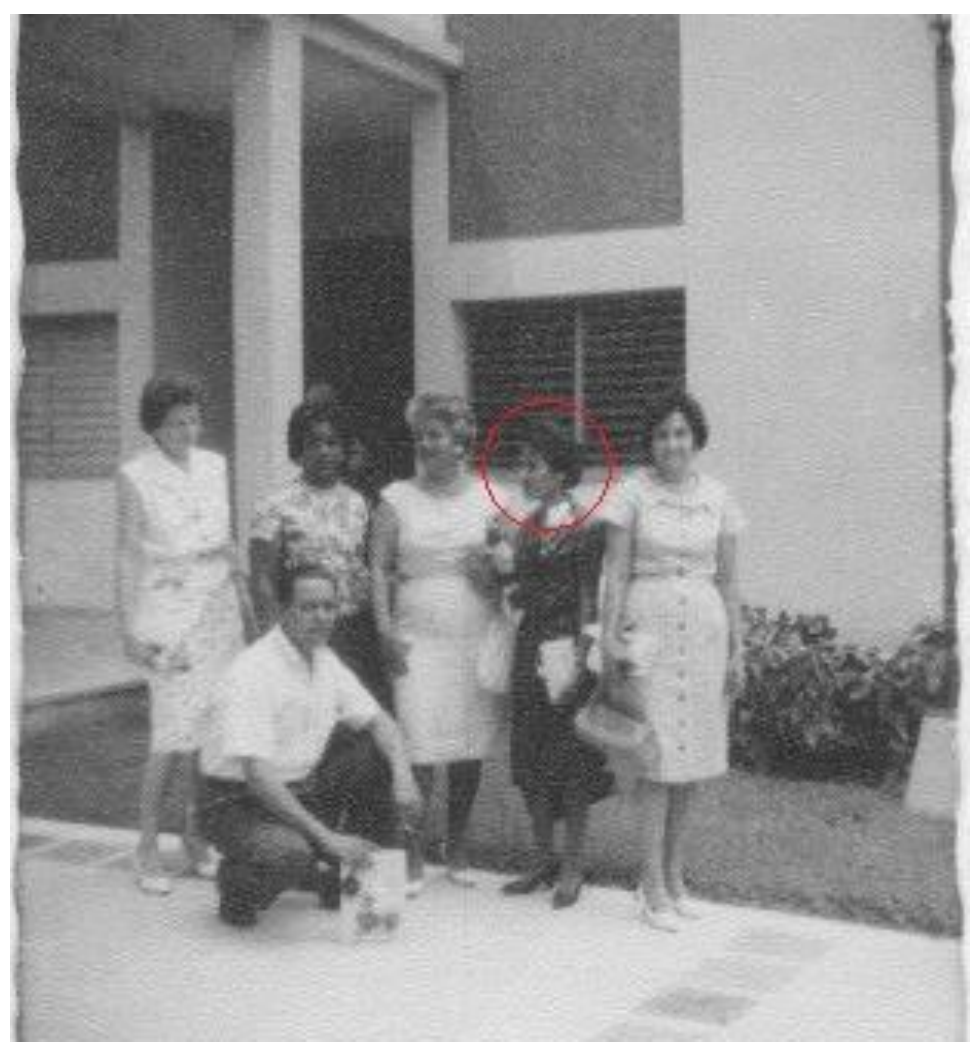

Ilustración 1: Olinta Ariosa Morales en la Escuela de Bibliotecarios: 1953.

Fuente: archivo personal de la familia de Olinta Ariosa. 
Sus primeros trabajos profesionales los realizó de forma voluntaria junto con otras compañeras de estudio, bajo la orientación del conocido bibliotecario argentino Carlos Víctor Penna (experto en bibliotecas del Centro Regional de la UNESCO para el hemisferio occidental, que residía en la ciudad de La Habana). Como resultado de esos esfuerzos, se pudo establecer una biblioteca escolar en la Escuela Primaria Superior No. 2 del Distrito Escolar de Marianao, que resultó ser pionera de esos servicios en Cuba. Olinta trabajó como bibliotecaria escolar, por una corta etapa en esta escuela de la provincia de La Habana. Ese mismo año regresó a Zulueta con su familia.

La recién fundada Universidad Central "Marta Abreu" de Las Villas en la región central de Cuba, convocó en junta general ordinaria el 21 de octubre de 1953, a través de su Consejo Universitario a desarrollar los ejercicios del Concurso-Oposición para cubrir la plaza de Estacionario de Biblioteca (Auxiliar). Como requisito exigido en la convocatoria librada por el Consejo Universitario estaba: ser graduado de Bibliotecario en la Universidad de La Habana. Olinta ya radicada en su provincia natal se presentó a los exámenes del concurso - oposición. Al respecto refiere Vivero, Rivera, Linares y Botana, (2007) que la noticia apareció en el número 4 de 1953 de la revista —Cuba Bibliotecológica:

....al quedar todavía vacantes dos plazas de auxiliar de bibliotecario se anunció nuevamente un concurso de oposición, consistentes en cinco ejercicios descritos. Al concurso se presentaron ocho personas y dos resultaron ganadoras: Olinta Ariosa obtuvo el primer lugar y Orlando Pariente, el segundo... (p. 32)

Dicho ejercicio a criterio de Manso (2014): "se desarrolló entre los días 27, 28 y 30 de octubre y el tribunal estuvo conformado por María Teresa Freyre de Andrade, como presidente, José M. Ruiz Miyar, como vocal, y como secretaria, Andrea Isora Pineda Cabrera" (p. 13). Los resultados de dicho ejercicio se aprueban en junta general ordinaria celebrada el 4 de noviembre de este propio año y por los Acuerdos 784 y 785 se adjudican dichas plazas a Olinta Ariosa Morales, quien obtuvo 65 puntos y a Mario Orlando Pariente Pérez, con una calificación de 62,50 puntos. En la propia junta del Consejo Universitario mencionado fue designada Marta Julia Edelmira Gómez Pino como Jefa de Auxiliares de Biblioteca, cargo que no desempeñó debido a la concesión de un año de excedencia en sus servicios. El Consejo Universitario designó en su lugar a Olinta Ariosa Morales y a Luisa Pacheco Rodríguez para el cargo de Auxiliar de Biblioteca. 
Olinta Ariosa se puede considerar como la primera directora de la naciente institución bibliotecaria de la Universidad Central "Marta Abreu" de Las Villas. En esta institución, Olinta desarrolló una incansable labor en aras del progreso de esta institución: sugirió la compra de equipos, mobiliarios, materiales documentales y elaboró el primer Reglamento con que constó la institución. Destacó la creación por parte de Olinta la presentación el 19 de abril de 1955 de la Memoria Anual de la Biblioteca General de 1954, donde se recoge el accionar de ese centro en cuanto a las adquisiciones realizadas, los donativos recibidos, la labor administrativa, los servicios prestados y sus estadísticas generales.

En la década de 1950 se estableció la celebración de las tres Jornadas Bibliotecológicas Cubanas, en 1953, 1954 y 1956. Dichos eventos significaron para los especialistas cubanos un importante punto de encuentro e interacción. Desde su primera edición del 5 al 18 de abril de 1953, estas fueron organizadas por la Asociación Nacional de Profesionales de Biblioteca, con el apoyo del Centro Regional de la UNESCO y de la Escuela de Bibliotecarios de la Universidad de La Habana.

Las Segundas Jornadas Bibliotecológicas Cubanas, se celebraron del 4 al 8 de mayo de 1954 en la ciudad de la Habana, siendo presidente de la Comisión Organizadora Fermín Peraza y Vicepresidenta María Teresa Freyre de Andrade. El tema central era el de las bibliotecas insertadas en los centros educacionales. En esta edición, el trabajo del evento estuvo enfocado hacia la reunión de los profesores y maestros para que, en estrecha colaboración con los bibliotecarios, tomaran parte en los debates que se produjeron alrededor de los trabajos de base. Así, se pretendía lograr un práctico intercambio de ideas en beneficio de maestros, bibliotecarios y alumnos para despertar el interés de los maestros hacia las actividades de la biblioteca.

Olinta en esta etapa, aunque era bibliotecaria de la Universidad Central "Marta Abreu" de Las Villas; tuvo inclinación por el estudio de las bibliotecas escolares (de nivel primario y bachillerato). En los eventos bibliotecológicos mencionados Olinta participó con el tema central de La Biblioteca en la Escuela Primaria. Presentó una ponencia titulada: "Función de la Biblioteca en la Escuela Elemental Moderna", destacando la importancia de crear 
bibliotecas en las escuelas primarias, así como interesar a la comunidad para solicitar del Estado la creación de Bibliotecas Escolares.

Las principales ideas de la ponencia fueron:

- Existen bibliotecas escolares en Cuba, pero no pueden considerarse como tales ya que la eficiencia de una biblioteca escolar está en su organización y las de Cuba carecen de ella.

- El estado actual de las bibliotecas escolares es de abandono y no desempeñan el papel que les reserva la educación.

- Los libros que forman esas bibliotecas no están seleccionados, dados los escasos medios de que dispone la escuela para adquirir libros y el descuido de las autoridades escolares al respecto.

- Las bibliotecas escolares como tales, sirviendo de ayuda al maestro en el desempeño de su labor educativa, pueden fomentarse en Cuba para situar nuestros métodos de enseñanza a la altura que merecen la mente despierta y ágil de nuestros muchachos.

- Debe interesarse a la comunidad para solicitar del Estado la creación de Bibliotecas Escolares.

Paralelamente iban desarrollándose sus actividades como bibliotecaria y fue desarrollando una obra literaria. En ese período surge el Boletín Revista del Colegio Nacional de Doctores en Ciencias y en Filosofía y Letras de La Habana. En dicho boletín Olinta publica la contribución "Las Rosas del Camino". El artículo de crítica literaria y corte biográfico trata sobre una poetisa poco conocida en Cuba. Su publicación se observa en la llustración 2. 


\section{R E V I S T A}

DEL COLEGIO NACIONAL DE DOCTORES EN CIENCIASS Y EN FILOSOFIA Y LETRAS

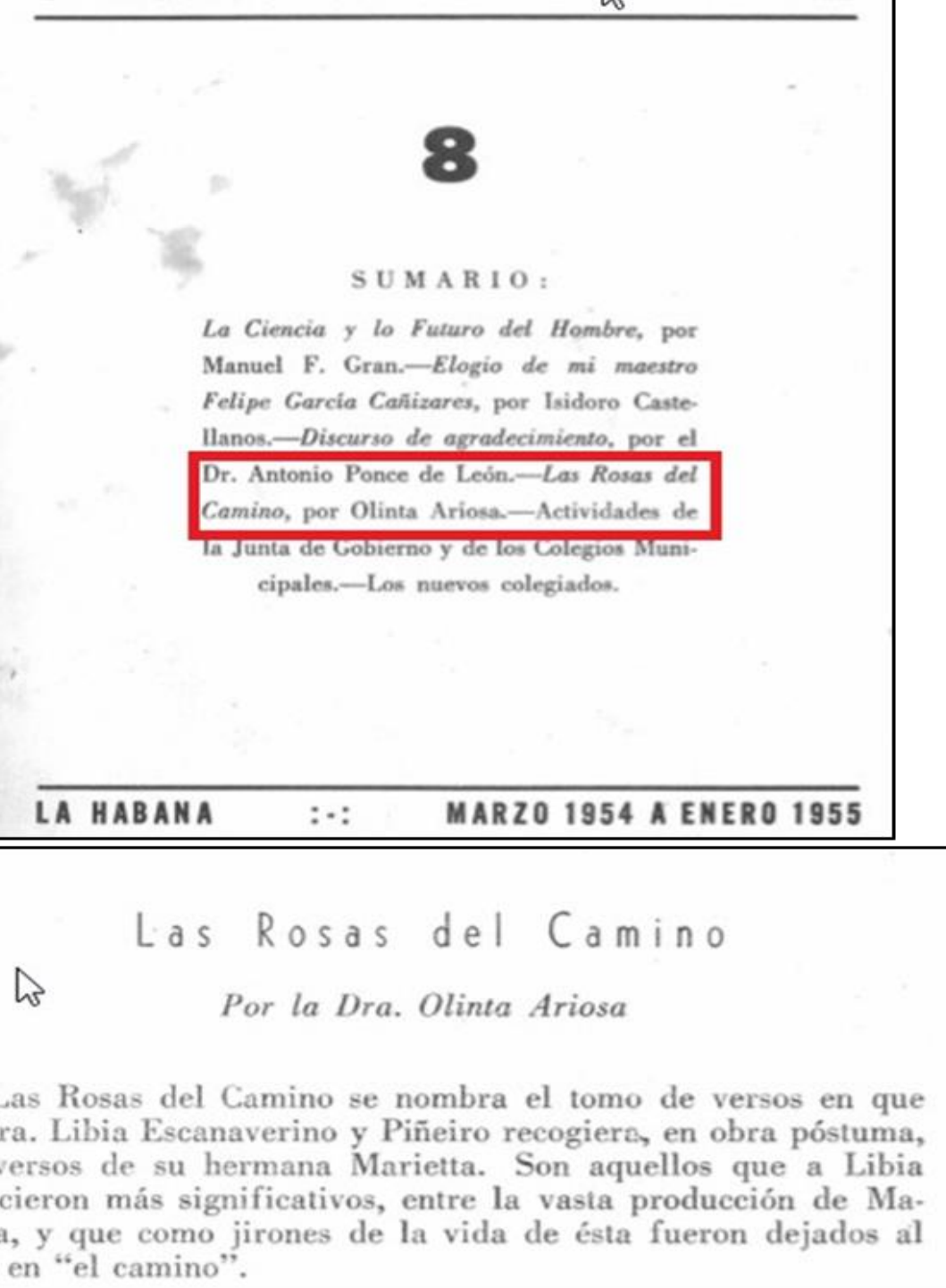

Ilustración 2: publicación del artículo Las Rosas en el Camino en la Boletín Revista del Colegio Nacional de Doctores en Ciencias y en Filosofía y Letras.

Fuente: Ariosa, 1954.

Su labor como bibliotecaria en la Biblioteca de la Universidad Central "Marta Abreu" de Las Villas quedó evidenciada en el Boletín Oficial de la Universidad del 1 de enero de 1955. En los acuerdos tomados por el Consejo Universitario, se hace referencia al permiso para adquirir, los libros solicitados por la doctora Olinta Ariosa Morales, Jefe de Auxiliares de la Biblioteca General. Se refiere además a la autorización de Olinta para que traslade al 
Bibliotecas. Vol. 39, № 2, julio - diciembre, 2021. EISSN: 1659-3286

URL: http://www.revistas.una.ac.cr/index.php/bibliotecas/index

Departamento de Extensión Cultural algunas obras, a fin de que por el mismo departamento se proceda a su correspondiente canje. Lo anterior se muestra en la llustración 3.

Seis del Presupuesto para el Ejercicio Econó- Universidad, los materiales que a continuación mico de 1954-1955. Lo que constituye parte de la unidad requerida para el Departamento AudioVisual, de la Universidad Central "Marta Abreu" de Las Villas, cuya compra se efectua a American Export Industries, Chicago, U.S.A para entregar C.I.F, La Habana, siendo dicho material de producción extranjera $y$ del que no se industrializa o fabrica en el Pais, resultando de esta forma más económico por obviarse los intermediarios vendedores nacionales $y$ extranjeros, obteniéndose de este modo que el precio sea fijo y uniforme, haciéndose imposible la competencia, lo que se acuerda al amparo de los artículos 55, inciso 2 de la Ley General de Contabilidad del Estado y 471, inciso 2, de la Ley Orgánica del Poder Ejecutivo",

ACUERDO TRESCIENTOS OCHENTA Y UNO: "Aprobar en todas sus partes el informe rendido por el Dr. José M. Ruiz Miyar, Profesor y Director de la Escuela de Filosofia y Letras de esta Universidad, sobre la visita a las distintas Universidades de Europa, a virtud de Acuerdo del Consejo Universitario".

Dr. Modesto de J, Pineda Cabrera, Secretario General.

Sesión ordinaria celebrada por el Consejo Económico el día 27 de Octubre de 1954.

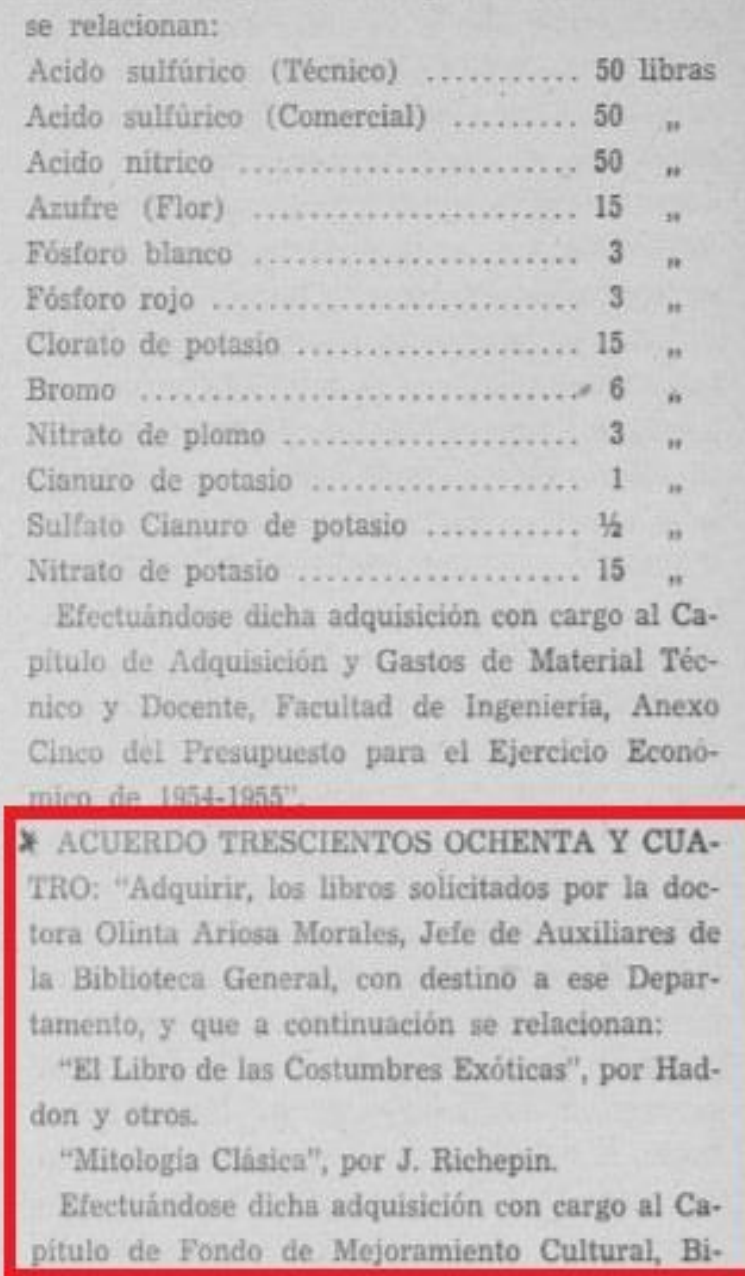

Ilustración 3: acuerdos del boletín oficial de la Universidad Central "Marta Abreu" de Las Villas donde se refiere Olinta Ariosa Morales.

Fuente: Universidad Central "Marta Abreu" de Las Villas, 1955.

En esta época circuló el periódico: El Mundo, en su edición del domingo 25 de septiembre de 1955 aparece una publicación de Olinta. Bajo el título "Surcando imágenes en la mente: los niños y los libros", la autora refiere a la importancia de la lectura para los niños.

Los libros, ilusiones, inquietudes, imaginación que corre en el espacio y hacia el espacio. Curiosidad infantil, que no se satisface ni se calma. Se agiganta. Acicate de 
la mente y definitivo, ansiedad. Eso y más representa un libro en manos de un niño. ¿Por qué se ha descuidado, entonces, el hábito de la lectura? Si los niños gustan y quieren leer... (Ariosa, 1955, p. 6)

En 1955 y 1956 se publicaron los artículos: "Peripecias en la reorganización de una biblioteca escolar" y "Función de la biblioteca en la Escuela Elemental Moderna", respectivamente. En el primero, la autora recomendaba cómo organizar una biblioteca escolar, hacer este trabajo ordenadamente, sin improvisaciones y con planes de desarrollo lógicos. El segundo fue presentado en las Segundas Jornadas Bibliotecológicas como una ponencia.

Su labor dentro de la biblioteca de la Universidad Central "Marta Abreu" de Las Villas la desempeñó hasta mayo de 1955, en que presenta su renuncia al ser designado un nuevo director para la Biblioteca. El nombramiento de Pacheco Rodríguez al cargo de Director de la Biblioteca, fue motivo de objeciones por parte de la dirección de la universidad. Este hecho tuvo una amplia repercusión, llegándose hasta a publicar un escrito en el diario El Pueblo, en su edición del 16 de mayo, donde se abordaba el desatino del Consejo Universitario al aprobar a Pacheco como director de la Biblioteca.

La renuncia de Olinta suscitó controversias dentro de la universidad, y el Consejo Universitario insta al Rector para que la persuada y retire su renuncia al puesto. Por ello a propuesta de una moción presentada por el Dr. Pedro Camps y Camps (primer rector de la universidad), se cita al Consejo Universitario para el 19 de mayo a junta general extraordinaria al efecto de tratar y resolver la renuncia presentada por Olinta y al mismo tiempo revocar el Acuerdo 916 en el que designaba a Pacheco Rodríguez como Director. La junta fue pospuesta en varias ocasiones, una vez efectuada no fue revocado el acuerdo por lo que Olinta abandona su cargo dentro de la biblioteca universitaria. Gran importancia en la vida de Olinta ocupaban las actividades políticas. En 1955 fue puesta en prisión por estar en desacuerdo con el gobierno. Se desconoce el tiempo que estuvo recluida, ya que las personas que ofrecieron testimonios no pudieron aportar sobre este particular y tampoco se encontró información documental. 


\section{b. Labor desarrollada como bibliotecaria y directiva de Olinta Ariosa Morales (1959-1999)}

En 1959 la Dr Olinta Ariosa Morales comienza a trabajar en la organización y puesta en marcha de la Biblioteca Municipal de Marianao. En 1962 es nombrada Directora del Departamento de Bibliotecas Escolares del Ministerio de Educación (ver llustración 1). Cuando se produjeron las primeras reformas en la enseñanza cubana Olinta creó las bibliotecas escolares para apoyar el proceso docente - educativo.

En el Departamento de Bibliotecas Escolares realizó una labor fundadora de esos servicios en Cuba, tanto en los aspectos administrativos como científico - metodológicos. Se rodeó de jóvenes profesionales y se convirtió en una maestra y formadora de cuadros. Este rasgo va a ser distintivo de su actividad durante el resto de su vida profesional. Olinta fue pionera en la aplicación en las bibliotecas cubanas de la Clasificación Decimal Dewey: un sistema práctico, con una notación simple, extensible y clara, fácil de aplicar y con margen para que en él pudieran caber los nuevos descubrimientos científicos que se realizaran. Era necesario para el procesamiento de los documentos en las bibliotecas escolares cubanas, una adaptación más práctica. Olinta incorporó al sistema de clasificación de las Bibliotecas Escolares, la clasificación decimal Melvin Dewey, este aporte apareció publicado en un folleto de impresión ligera en el año 1962 (ver llustración 4).

Sobre la labor de Olinta en la época refirió el Dr. Emilio Setién Quesada: conocí a Olinta en los años 60 cuando dirigía el Departamento de Bibliotecas Escolares del Ministerio de Educación (MINED), coincidimos en distintas actividades del sector. Hasta 1965 ella impartió clases de Bibliología en la Escuela de Bibliotecarios de la Universidad de La Habana. Llegué a ser su alumno cuando en esa década cursé los estudios de Bibliotecología en la Universidad de La Habana.

En 1967 la Dra. Olinta Ariosa y el Dr. Orlando Pariente impartieron un postgrado sobre Metodología de la Investigación Bibliográfica en la Universidad Central "Marta Abreu" de Las Villas. El periodista Jesús Dueñas Becerra atestigua la significación del postgrado y los recuerdos que guarda de sus enseñanzas: a los doctores Olinta Ariosa Morales y Orlando Pariente Pérez los conocí como profesores de un postgrado sobre Metodología de la Investigación Bibliográfica, impartido en la Universidad Central en 1967 y al que asistí 
como requisito sine qua non para comenzar mi adiestramiento como profesor de Metodología de la Investigación en la Facultad de Humanidades de mi querida Alma Mater. Su larga trayectoria como bibliotecaria estuvo siempre ligada a la pasión que profesaba por la lectura y por los libros, a lo que sumaba una poderosa vocación de servicio. Solo una persona tan entregada a los nobles menesteres de ayudar, tanto en las búsquedas más difíciles y apremiantes, como en la orientación adecuada, podía llegar a ser la profesional que resultó Olinta.

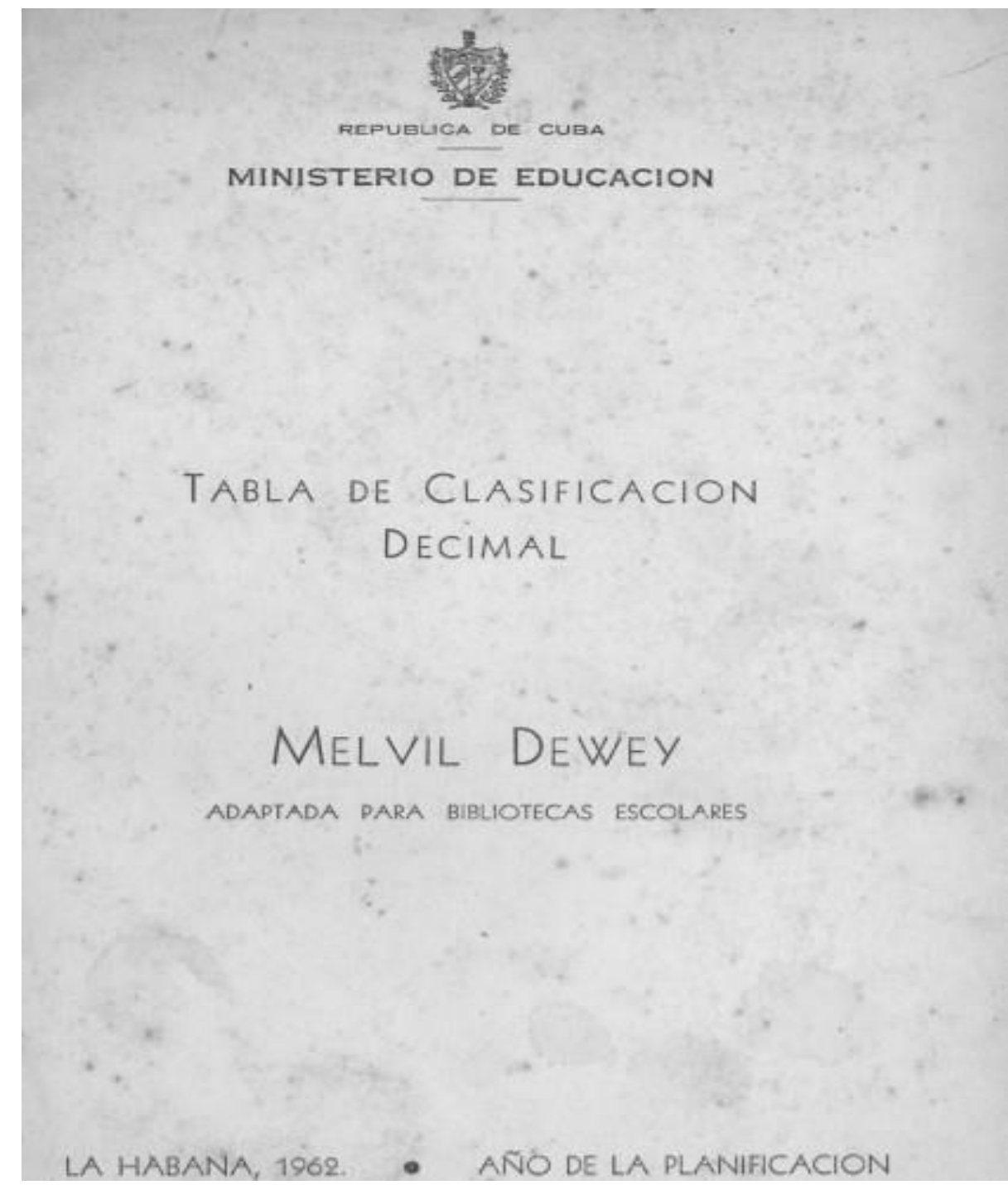

Ilustración 4: acuerdos del boletín oficial de la Universidad Central "Marta Abreu" de Las Villas donde se refiere Olinta Ariosa Morales.

Fuente: Ariosa, 1962. 
Pasó a la Dirección del Instituto de Documentación e Información Científico Técnica (IDICT) de la Academia de Ciencias de Cuba en 1972. Su labor allí se destacó por haber echado los cimientos de la modernización de los servicios de información en Cuba, con la introducción de la enseñanza de las nuevas técnicas de procesamiento, la adopción de lenguajes informativos, la publicación de textos y los primeros intercambios con bibliotecarios de otros países.

Del 14 al 16 de mayo de 1972, se celebró en el Hemiciclo² de la Academia de Ciencias de Cuba el I Fórum sobre Literatura Infantil y Juvenil. Olinta presentó la ponencia titulada "La literatura científica y la juventud cubana". Siendo muy joven Olivia E. Ferrán Lopetegui trabajó junto a Olinta, sobre su colega expone: me llamó para hablar, se sentó en su buró siendo jefa de la Biblioteca del IDICT, me aconsejó por mi juventud para que siguiera estudiando, diciéndome: "papelitos a la lengua". Fue preocupación constante de Olinta la superación de sus trabajadores, especialmente de los jóvenes. Mantuvo magníficas relaciones de trabajo con todos. En este tiempo se desempeñó como directora del IDICT y como jefa del Departamento de la Biblioteca, ambos cargos los desempeñó simultáneamente. Olivia agregó: era buena directora, carácter afable, buena compañera, muy humana, buena con todo el mundo, a pesar de que era muy recta y disciplinada para el trabajo.

Al crearse el Ministerio de Cultura en 1976, asumió la Dirección Nacional de Bibliotecas cuyo objetivo de trabajo era el desarrollo científico metodológico de las Bibliotecas Públicas del país. Entre 1976 - 1977 la Dra. Olinta Ariosa Morales es Directora interina de la Biblioteca Nacional "José Marti" (BNJM), le sucede a Luis Suardíaz (1973 - 1976) y le precede a Julio Le Riverend Brusone (1977 - 1988). La doctora Olinta Ariosa se había destacado también antes de 1959 por su labor en pro de las bibliotecas públicas, sin embargo, no fue de las que integró el equipo de trabajo formado por la doctora Freyre de Andrade desde la Biblioteca Nacional.

El período de María Teresa Freyre de Andrade acaeció desde 1959 - 1967, en esta etapa Olinta realizó meritoriamente otras tareas importantes dentro de las bibliotecas cubanas. Sobre su período como directora de la Biblioteca Nacional refiere Emilio Setién: Olinta fue designada por el propio Ministro de Cultura de entonces, el Doctor Armando Hart, con 
quien había trabajado ya en el MINED cuando él fue Ministro de Educación; le encargó la organización de las Bibliotecas Escolares. Su aval para el cargo en la BNJM viene dado, precisamente, por su actividad en Bibliotecas Escolares, por haber dirigido también el IDICT de la Academia de Ciencias y por haber sido profesora de Bibliotecología en la Universidad de La Habana.

Fernández (2001) establece: "la presencia de la doctora Olinta Ariosa al frente de la Dirección Nacional de Bibliotecas no ha sido subrayada en toda su dimensión, pues después de la doctora Freyre de Andrade no hubo, un verdadero bibliotecólogo a ese nivel" (p. 47). En estos años Olinta pudo apreciar la necesidad de la BNJM de una nueva estructura acorde con el desarrollo profesional alcanzado. Desde la Dirección Nacional de Bibliotecas Públicas potenció un enfoque sistémico a esa actividad, perfeccionando la estructura de la base administrativa. La estructura sugerida por ella fue aplicada por el Dr. Julio Le Riverend Brusone al ocupar la Dirección de la Biblioteca Nacional a partir del 2 de diciembre de 1977. Al poco tiempo de ocupar tan alto puesto, Le Riverend reorganizó la institución en los siguientes departamentos sugeridos por Olinta: Investigaciones Bibliotecológicas y Metodológicas, Selección y Adquisición, Procesamiento Técnico, Fondos Bibliográficos, Servicios al Público, Investigaciones Histórico - Culturales, Ediciones y Conservación, Juvenil, Investigaciones Bibliográficas, Información para la Cultura y el Arte, Circulante Extensión Bibliotecaria, Economía, Personal, Cuadros y Capacitación, Mantenimiento y Servicios Generales. Una imagen de Olinta correspondiente a ese período se muestra en la llustración 5 .

Viciedo (2006) al referirse a Olinta en el período comprendido entre 1977 y 1985, establece que: fue un período saludable para la biblioteca pública cubana que, bajo la orientación de Olinta Ariosa, desarrolló un conjunto de actividades profesionales que mejoraron el panorama de este sector desde el punto de vista científico y técnico. Ejemplo de lo anterior lo constituye la creación de las Coordinaciones Provinciales de Bibliotecas en las 14 provincias y el municipio especial Isla de la Juventud. Estas coordinaciones fueron los órganos cabeceras de las bibliotecas públicas en cada provincia cuyo órgano rector era la Dirección Nacional de Bibliotecas. 


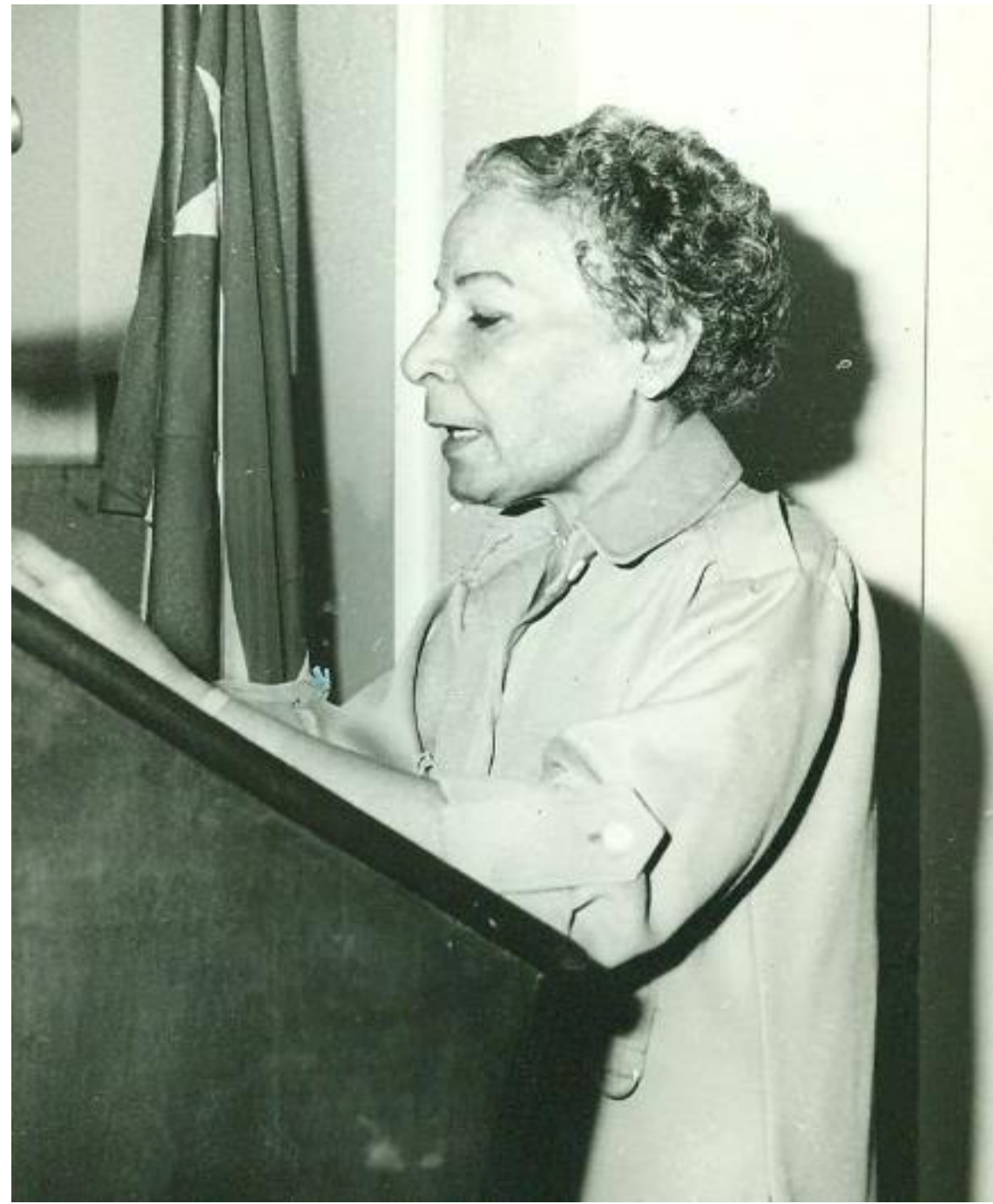

Ilustración 5: Olinta Ariosa Morales en la Biblioteca Nacional "José Marti”" de Cuba. Fuente: fondo fotográfico de la familia de Olinta Ariosa.

Con la dirección de Olinta se iniciaron institucionalmente las investigaciones de carácter científico en la esfera de la bibliotecología en el país. La investigación tomó cuerpo y se convirtió en un programa nacional ramal, en un programa local de las bibliotecas o de la Biblioteca Nacional, particularmente. Se organizaron temas de investigación de carácter nacional con la participación de las bibliotecas públicas. Especialistas integrados en equipos temáticos dirigieron y siguieron el desarrollo de las investigaciones, así como de la introducción de sus resultados. Como resultado de esta labor científica, un reducido grupo de especialistas se categorizó como investigadores a instancias del Centro de Investigaciones para la Cultura Cubana "Juan Marinello" del Ministerio de Cultura, entre los cuales puede citarse a Emilio Setién y Zoila Lapique. 
A partir de la labor científica realizada con el concurso de varios especialistas, se creó el "Registro Primario de Información", conocido entre los bibliotecarios públicos como los BP (biblioteca pública). Era un sistema de modelos de control estadístico para la recogida y procesamiento de la información primaria por medio del cual las bibliotecas podían controlar toda la actividad bibliotecaria de manera inmediata. Con los modelos diseñados se pudo obtener información sobre el incremento del fondo bibliotecario, los tipos de documentos existentes, la demanda insatisfecha, los tipos de préstamos, la circulación y rotación del fondo, así como otros datos de interés. Esta información, clasificada, permitió al bibliotecario no solo conocer el comportamiento estadístico de los servicios y del fondo de su institución, sino también la realización de estudios bibliotecológicos sobre el funcionamiento de la biblioteca.

Olinta fue responsable de la revisión técnica y de la redacción de la nota de la edición cubana del libro "Bibliotecología General" de Chubarian (1976) (ver Ilustración 6). En el prólogo Olinta expone la importancia del documento para los profesores universitarios de Bibliotecología y de las escuelas técnicas del país. Era un texto de apoyo a la docencia y una de las primeras ediciones de este tipo de material en el país.

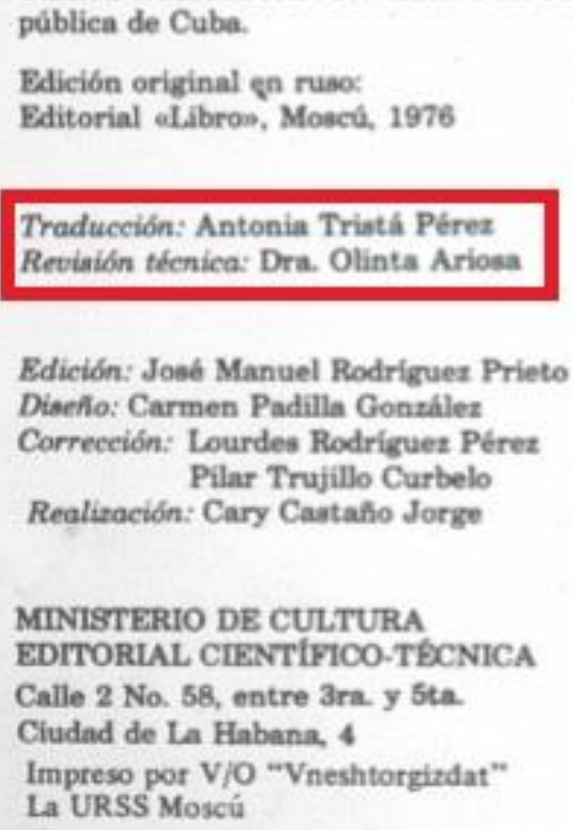

Ilustración 6: edición cubana del libro "Bibliotecología General" donde Olinta Ariosa realiza la revisión técnica.

Fuente: Chubarian (1976). 
Durante su dirección en la Biblioteca Pública se formaron los primeros cubanos como candidatos a doctores en la especialidad de Bibliotecología y Bibliografía. Se incorporaron nuevos enfoques de los procesamientos bibliotecológicos, así como de los servicios entre lo que se destacan las minibibliotecas. Se crearon las investigaciones de la actividad bibliotecológica y se fortalecen los encuentros y Jornadas Bibliotecarias (desde el nivel municipal, provincial y nacional). La llustración 7 muestra un encuentro bibliotecológico nacional realizado en 1984.

Sobre el aspecto anterior Emilio Setién refiere: Olinta apoyó e impulsó todo el movimiento de investigaciones de esa índole en el sistema de bibliotecas públicas durante su dirección. Fue la época en que se efectuaron los eventos científicos nacionales de la profesión, se constituyeron los primeros problemas ramales propios, se otorgaron categorías científicas a los bibliotecarios y se hicieron los primeros doctorados por el personal del sistema de bibliotecas públicas.

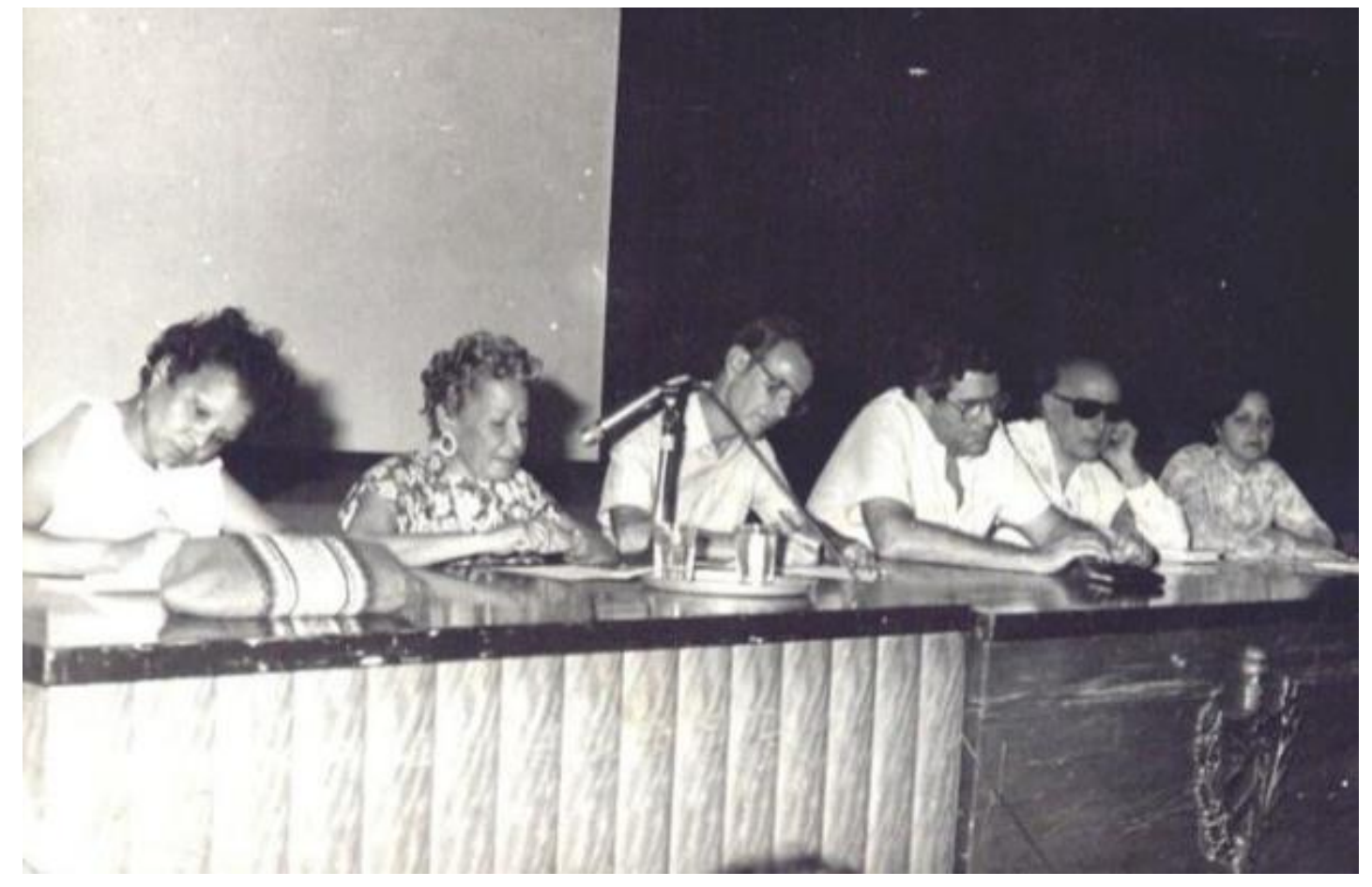

Ilustración 7: presidencia de la Jornada Bibliotecológica Nacional, en la fotografía Olinta Ariosa se encuentra más cercada al micrófono, a su izquierda se encuentra Emilio Setién Quesada.

Fuente: fondos fotográficos de la Biblioteca Nacional "José Marti" de Cuba. 
Durante el período de Olinta se introdujo el concepto de las diez instituciones culturales en cada municipio del país, entre las que la biblioteca era pilar básico. Olinta fue iniciadora e introductora de la alfabetización en Braille para los ciegos y débiles visuales, a través de la creación de salas y servicios especializados para este tipo de usuario en las Bibliotecas Públicas del país. Lo anterior se llevó a cabo con la coordinación entre la Asociación Nacional de Ciegos (ANCl) y la Dirección del Sistema de Bibliotecas Públicas del país. Se establecieron las directrices para la creación de servicios bibliotecarios para ciegos en conjunto con la biblioteca provincial de La Habana "Roberto García Valdés" y se inaugura la primera área especial para ciegos y débiles visuales, así como un aula de alfabetización en 1979.

Olinta trabajó para la representación de Cuba en la Federación Internacional de Asociaciones de Bibliotecas (IFLA). La participación de los profesionales cubanos en las conferencias y congresos generales de IFLA prestigió la bibliotecología cubana. Lo anterior permitió ubicar a las bibliotecas públicas en el contexto internacional del mundo de la profesión, a la vez que permitió medir el desarrollo alcanzado por estas instituciones con el de otras naciones de extensa tradición bibliotecaria.

En los primeros años de la década del 80, Olinta presidió la primera delegación de la Conferencia de IFLA, asistiendo en compañía de otros bibliotecarios cubanos. Algunos de estos especialistas formaron parte de las diferentes secciones profesionales como miembros correspondientes en teoría bibliotecaria, libros raros y valiosos, bibliotecas infantiles y bibliografía, entre otras. La opinión internacional con respecto a los bibliotecarios cubanos tuvo un cambio incidido por la labor científica desarrollada por esos profesionales. Olinta Ariosa participó en seis Congresos de IFLA: 1) 1980 Filipinas, 2) 1981 Leipzig, 3) 1982 Montreal, 4) 1983 Monechs, 5) 1984 Nairobi y 6) 1985 Chicago.

Se crearon en esta etapa grupos metodológicos para el asesoramiento al trabajo profesional, orientarían, a su vez, la labor metodológica bibliotecaria en sus respectivas provincias. Al equipo, se incorporaron otros especialistas que también contribuyeron a la continuidad del desarrollo del trabajo bibliotecario para el Sistema Nacional de Bibliotecas. Especialistas como Emilio Setién, Miriam Bendamio, Laura Rumbau, Concepción Lorenzo, Dania Salvat, Norma Avalos, Cila Delia Pentón y la propia Olinta Ariosa fueron 
responsables de la asesoría y orientación metodológica del sistema de Bibliotecas Públicas.

Concepción Lorenzo Hernández más conocida como Conchita, recuerda sobre esta etapa con Olinta: nos conocimos en agosto de 1980 en el Ministerio de Cultura, a través de otra compañera amiga mía. Olinta me entrevistó para ocupar una plaza de especialista en el Departamento Metodológico. En diciembre del propio año la Dirección de Bibliotecas Públicas traslada el Departamento Metodológico de Bibliotecas Públicas para F y 15 en el Vedado, La Habana. En aquel entonces, el departamento se dividía a su vez en dos áreas: 1) Superación y Cuadros y 2) Departamento de Sistemas, en el cual soy jefa por sustitución durante un año. Olinta tenía buenas relaciones con los subalternos, nos hablaba pausado, oía a todo el mundo, éramos un equipo de trabajo pequeño, pero un buen equipo de trabajo.

Olinta confiaba mucho en nosotros, nos daba libertad de acción, les decía a sus subalternos que escribieran para que no se perdieran sus trabajos. Fue ejemplo para todos porque fue una disciplina laboral viviente. Ella llegaba muy temprano al trabajo y era la última en irse. Para mí, es un ejemplo de profesional: laboriosa, sencilla y humilde que dio todo de sí, sin esperar recompensas, siempre ayudando a los demás sobre todo a los jóvenes, repartiendo sabiduría y profesionalidad por donde pasara. No permitió que la diabetes entorpeciera con su labor, un día en una reunión del Ministerio cayó al suelo y no permitió que se suspendiera la reunión, ella era así, lo daba todo.

Olinta Ariosa Morales se empeñó en revitalizar la Asociación Cubana de Bibliotecarios $(\mathrm{ASCUBI})^{3}$, siendo la primera presidenta de la directiva en su fundación el 31 de marzo de 1986 (día del libro cubano). Como vicepresidenta primera se desempeñó la Dra. Marta Terry González y como vicepresidentas: Miriam Martínez Crespo y Adelina López Llerandi. La secretaria de organización fue Blanca Mercedes Mesa. Por su meritoria labor como bibliotecaria y directiva, ostentó varias distinciones y galardones como:

- Sello Conmemorativo "Antonio Bachiller y Morales": entregado por primera vez en acto central celebrado el 7 de junio de 1995 por el día del bibliotecario cubano. Organizado por la ASCUBI y la Sociedad Cubana de Ciencias de la Información (SOCICT) en el Salón de los Pasos Perdidos del Capitolio Nacional. 
- Distinción por la Cultura Cubana que otorga el Ministerio de Cultura.

- Orden Alejo Carpentier en 1983 por su abnegada labor cultural.

Olinta trabajó hasta el año 1989. Los últimos años de su vida, Olinta los vivió junto a su hermano menor, Julio y la familia creada por este en la casa de Ayestarán \# 120 ciudad de La Habana. Murió en agosto de 1999 a los 78 años. En reconocimiento de sus méritos y su trabajo en beneficio de las bibliotecas escolares, la ASCUBI tomó la decisión de instaurar a partir del 14 de abril de 2004, entre otros premios de la profesión, el "Premio Nacional Olinta Ariosa Morales". Se otorgará a los bibliotecarios escolares, miembros de la ASCUBI - SOCIT que en su quehacer se destaquen por la adhesión a los principios éticos de ambas asociaciones y por su labor en favor de la promoción de la lectura, del conocimiento y la investigación como apoyo a la labor docente educativa. También fue instaurado el 14 de abril como Día del Bibliotecario Escolar Cubano en honor al natalicio de Olinta Ariosa.

\section{Conclusiones}

Las investigaciones históricas en el ámbito bibliotecológico-informativo, contribuyen a fortalecer la identidad de los miembros del gremio. Lo anterior permite además identificar personalidades que prestigian la labor del sector. Las historias de vida son una vía para el análisis exhaustivo de la labor de importantes bibliotecarios en el ámbito de Cuba.

El desarrollo científico de la Bibliotecología en Cuba revela la existencia de un gran número de bibliotecólogos y educadores; con sus incuestionables aportes teóricos, prácticos y metodológicos facilitaron el surgimiento y desarrollo de la Bibliotecología como ciencia, dándole el sólido prestigio con que hoy cuenta el perfil bibliotecológico. El estudio realizado es muestra de la importancia de los estudios históricos y su extensión a otras personalidades de la bibliotecología cubana.

Las contribuciones fundamentales de Olinta Ariosa a la Bibliotecología cubana están referidas, necesariamente a experiencias concretas en su labor como bibliotecaria y dirigente del Sistema de Biblioteca Escolar y Bibliotecas Públicas. Fungió como Directora interina de la Biblioteca Nacional "José Marti". Fue iniciadora e introductora de la alfabetización en Braille para los ciegos y débiles visuales. Representó a Cuba en varias ocasiones en la Conferencia de IFLA. Fue la primera presidenta de la directiva de ASCUBI. 
En honor a toda su labor en el ámbito de la bibliotecología se instaura en Cuba el 14 de abril de como Día del Bibliotecario Escolar en honor al nacimiento de Olinta y en 2004 se crea el "Premio Nacional Olinta Ariosa Morales" para reconocer a los bibliotecarios escolares.

\section{Referencias bibliográficas}

Archivo personal de la familia Ariosa. (1953). Fotografía de Olinta Ariosa Morales en la Escuela de Bibliotecarios.

Archivo personal de la familia Ariosa. (1977). Fotografía de Olinta Ariosa Morales en la Biblioteca Nacional "José Martî" de Cuba.

Ariosa, O. (1954). Las Rosas del Camino. Boletín Revista del Colegio Nacional de Doctores en Ciencias y en Filosofía y Letras, 4(2), 22-24.

Ariosa, O. (1955). Surcando imágenes en la mente. Los niños y los libros científicos, El Mundo, Comentario, p. 4.

Ariosa, O. (1962). Tabla de clasificación decimal Melvin Dewey adaptada para bibliotecas escolares. La Habana: Ministerio de Educación.

Biblioteca Nacional "José Marti" de Cuba. (1984). Fondo fotográfico colección eventos: 1980 - 1990. Presidencia de la Jornada Bibliotecológica Nacional en 1984.

Chubarian, O. S. (1976). Bibliotecología general. La Habana: Ministerio de Cultura. Editorial Científico Técnico.

Fernández, T. (2001). Apuntes para la historia de la Biblioteca Nacional "José Martí" de Cuba. La Habana: Biblioteca Nacional de Cuba.

Frías, M., Paz, L. E., Alomá, C., Tamayo, D., y Hernández, E. A. (2019). El boletín ASCUBI Villa Clara, reflejo del acontecer informativo en el centro de Cuba. Bibliotecas: Revista de la Escuela de Bibliotecología, Documentación e Información, 37(1), 1-23. Manso, R.A. (2014). Historia de la Biblioteca General de la Universidad Central «Marta Abreu» de Las Villas (1952 - 1959). Santa Clara: Editorial Feijóo.

Montes de Oca, D. (2005). María Teresa Freyre de Andrade, precursora de la Bibliotecología moderna. (Tesis presentada para optar por el título de Doctor de Ciencias de la Información), Universidad de La Habana, La Habana.

Paz, L. E. (2015). Reportes de Información: oferta de información web para la filial de la Asociación Cubana de Bibliotecarios en Villa Clara. (Trabajo de diploma presentado 
para optar por el título de Licenciado en Ciencias de la Información), Universidad Central "Marta Abreu" de Las Villas, Santa Clara.

Paz, L. E. (2018). Actividad editorial y socialización de la ciencia. Santa Clara: Editorial Feijóo.

Paz, L. E., Hernández, E. A., Tamayo, D., y Frías, M. (2016). El diseño de ofertas de información basado en la experiencia de usuarios. El caso del boletín Reportes de Información. Métodos de información, 7(12), 89-116.

Rivera, Z., y Silva, A. (2006). José Antonio Ramos, el autor del Manual de biblioeconomía y uno de los más ilustres intelectuales de la República en Cuba. Acimed, 14(6), 1-18.

Rivera, Z., y Silva, A. (2007). Fermín Peraza Sarausa: bibliógrafo y bibliotecólogo. Acimed, 15(1), 1-14.

Rodríguez, M., y Rivera, Z. (2006). María Villar Buceta: la primera profesora de biblioteconomía en Cuba. Acimed, 14(2), 1-23.

Silva, A., y Rivera, Z. (2006). Domingo Figarola Caneda: una personalidad de la cultura y la bibliotecología en Cuba. Acimed, 14(2), 1-16.

Terry, M. (2004). La mujer bibliotecaria cubana en la sociedad de hoy. Ponencia presentada en el I Foro Social de Información, Documentación y Bibliotecas, Buenos Aires.

Universidad Central "Marta Abreu" de Las Villas. (1955). Acuerdos y resoluciones. Boletín Oficial de la Universidad Central "Marta Abreu" de Las Villas, 1955(1), 144-155.

Viciedo, M. (2006). La biblioteca pública cubana en el período 1959-1989. Acimed, 14(3), $1-13$.

Vivero, N., Rivera, Z., Linares, R., y Botana, M. (2007). La revista Cuba Bibliotecológica: reflejo del desarrollo de la bibliotecología cubana en la década de los años 1950. Acimed, 16(6), 1-54.

\section{Notas de las autorías}

Miriam Caridad Ruíz García. Máster en Psicopedagogía. Profesora asistente del Departamento de Ciencias de la Información. Universidad Central "Marta Abreu" de Las Villas, Cuba. Correo electrónico: miriamr@uclv.edu.cu ORCID: https://orcid.org/0000-0002$\underline{5309-6160}$ 
Luis Ernesto Paz Enrique. Licenciado en Ciencias de la Información. Profesor asistente del Departamento de Ciencias de la Información. Editorial Universitaria Samuel Feijóo. Universidad Central "Marta Abreu" de Las Villas, Cuba. Correo electrónico: luisernestope@uclv.cu ORCID: https://orcid.org/0000-0001-9214-3057

Cándida Judith Guerra Miranda. Licenciada en Bibliotecología. Biblioteca Provincial "Marti" de Villa Clara. Correo electrónico: cjudith@nauta.cu ORCID: https://orcid.org/0000-0002$\underline{6150-8583}$

${ }^{1}$ A partir de 1976 esta provincia se divide en tres: Cienfuegos, Villa Clara y Sancti Spíritus. El poblado de Zulueta se localiza en la provincia de Villa Clara.

2 Refiere a una parte de la antigua construcción que ocupaban el Instituto de Documentación e Información Científico Técnica de la Academia de Ciencias de Cuba. Actualmente el Capitolio de La Habana constituye la sede del gobierno.

${ }^{3}$ Cabe destacar que luego de 1959 los bibliotecarios no contaban con una asociación profesional que los aglutinara, no es hasta 1980 que surge la Asociación Cubana de Bibliotecarios con las siglas ASCUBI. Anterior a 1959 existieron varias asociaciones de profesionales de la información, de las cuales la de mayor prestigio y reconocimiento fue la denominada Asociación Cubana de Bibliotecarios con las siglas ACB. 